\title{
Sensitive Quantitation of Residual Phenylhydrazine in Antipyrine by LC-ICP- MS with Iodo Derivatization
}

\author{
Koki Harigaya, ${ }^{* \dagger}$ Hiroyuki Yamada, ${ }^{*}$ Shingo Horimoto, ${ }^{*}$ Hiroyuki NishI,** and Jun Haginaka*** \\ *Analytical Research Laboratories, CMC Division, Mitsubishi Tanabe Pharma Corp., 3-16-89 Kashima, \\ Yodogawa, Osaka 532-8505, Japan \\ **Department of Pharmaceutical Chemistry, Faculty of Pharmacy, Yasuda Women's University, \\ 6-13-1 Yasuhigashi, Asaminami, Hiroshima 731-0153, Japan \\ ***School of Pharmacy and Pharmaceutical Sciences, Mukogawa Women's University, 11-68 Koshien \\ Kyuban-cho, Nishinomiya 663-8179, Japan
}

\begin{abstract}
Phenylhydrazine is a genotoxic impurity commonly used as a raw material in active pharmaceutical ingredients. In this study, a novel method, using inductively coupled plasma-mass spectrometry combined with two-dimensional liquid chromatography (LC-ICP-MS), was employed for the quantitation of residual phenylhydrazine in antipyrine. The compound 2,3,5-triiodobenzoyl chloride, which contains three iodine atoms, was investigated as a derivatization reagent. The DL $0.06 \mathrm{ppm}$ was obtained, and the method was applied to the quantitation of residual phenylhydrazine in antipyrine. No residual phenylhydrazine was detected in five lots of antipyrine obtained from the commercial source.
\end{abstract}

Keywords Phenylhydrazine, LC-ICP-MS, 3-iodophenyl isocyanate, 2,3,5-triiodobenzoyl chloride, genotoxic impurity, antipyrine, active pharmaceutical ingredient

(Received May 9, 2014; Accepted June 23, 2014; Published August 10, 2014)

\section{Introduction}

During the development of pharmaceutical products, residual impurities such as raw materials, intermediates, by-products, excipients, and degradation products are controlled according to the International Conference on Harmonization (ICH) Q3A/ Q3B guidelines. ${ }^{1}$ Control of the residual genotoxic impurities (GTIs) is one of the critical concerns in the development of active pharmaceutical ingredients (APIs). However, the toxicological assessment of GTIs is not addressed in sufficient detail in the ICH Q3A/Q3B guidelines. Recently, the ICH M7 regarding the limits of GTIs was discussed and is now under an ICH consensus process. The US Food and Drug Administration (FDA) and European Medicines Agency (EMA) have published guidelines (for the control of GTIs). ${ }^{2-5}$ These agencies set the threshold of toxicological concern at $1.5 \mu \mathrm{g} /$ day for GTIs in APIs or drug products. Thus, the allowable limits of GTIs are generally at ppm levels, depending on the duration and daily exposure dose. The risk assessment of GTIs during clinical development has become a major liability. ${ }^{6-10}$ Therefore, in addition to the rigid manufacturing process controls, a sensitive and selective analytical method for the detection of GTIs at low ppm levels is required. ${ }^{11-13}$

Phenylhydrazine is one of the GTIs commonly used as a raw

$\dagger$ To whom correspondence should be addressed.

E-mail: harigaya.koki@mc.mt-pharma.co.jp

K. H. present address: Technology Department, Yoshitomi Plant, Mitsubishi Tanabe Pharma Factory Ltd., 955 Koiwai, Yoshitomi, Chikujo, Fukuoka 871-8550, Japan. material for APIs. Recently, the use of phenylhydrazine in API synthesis has been avoided because of its genotoxicity. ${ }^{10}$ However, if it is unavoidable to use phenylhydrazine during the manufacturing of an API, an additional process step such as purification may be required to reduce the amount of phenylhydrazine in the APIs. In that case, the residual amount of phenylhydrazine in the final API must be monitored. Therefore, a sensitive and selective analytical method is required to confirm the amount of phenylhydrazine in the starting and intermediate materials and/or API to ensure compliance with safety needs.

Generally, liquid chromatography-mass spectrometry (LC-MS) and gas chromatography-mass spectrometry (GC-MS) have been used for trace level analyses of GTIs in APIs. ${ }^{14-20}$ However, in some cases, these techniques might not be applicable because of the properties (ionization efficiency, polarity, solubility, and purity, etc.) of the GTIs and/or APIs. To overcome this problem, it is preferable to have an alternative methodology for GTI analysis. Inductively coupled plasma-mass spectrometry (ICP-MS), which has been utilized for trace level elemental analysis in many industries, is one of the options for trace analysis because of the high sensitivity of the system. ${ }^{21-23}$ Applications of ICP-MS to the control of residual metals and speciation studies concerning several metals in APIs have been successfully performed. ${ }^{24,25}$ Moreover, ICP-MS has been used for organic analyses because of its highly efficient ICP ionization and selective, sensitive MS detection. ${ }^{26,27}$ Based on these perspectives, we have developed a novel analytical method for one GTI, 4-chloro-1-butanol, using ICP-MS with iodo derivatization. ${ }^{28}$ The use of iodine for sensitive detection by ICP-MS is advantageous because of the low background, which 
is a result of the slight interference of polyatomic ions at $\mathrm{m} / \mathrm{z}$ 127 (iodine). The developed method can also be applied to other GTIs such as phenylhydrazine.

In this study, an LC-ICP-MS method with iodo derivatization for the analysis of residual phenylhydrazine in APIs was investigated. First, since phenylhydrazine has no sensitive element for an ICP-MS detector, the mono-iodo derivatization reagent (3-iodophenyl isocyanate) was utilized for the detection of phenylhydrazine. In the previous study, 3-iodobenzoyl chloride was utilized as an iodo derivatization reagent, and it can be applied to phenylhydrazine analysis. ${ }^{28}$ However, in order to expand the range of options of the iodo derivatization reagents, 3-iodophenyl isocyanate was investigated. Incidentally, sensitivity for an ICP-MS analysis is considered to be almost the same between the reagents because both reagents have one iodine atom in the structure. Then, the tri-iodo derivatization reagent (2,3,5-triiodobenzoyl chloride) was utilized with the expectation that it would improve sensitivity. The detection limit (DL) obtained from the two reagents was evaluated with regard to the number of iodine atoms. Finally, since phenylhydrazine is widely used as a raw material for antipyrine, the developed LC-ICP-MS methods with 3-iodophenyl isocyanate or 2,3,5-triiodobenzoyl chloride were applied for the quantitation of residual phenylhydrazine in antipyrine.

\section{Experimental}

\section{Chemicals and reagents}

Purified water $(18.2 \mathrm{M} \Omega \cdot \mathrm{cm})$ from a Milli-Q water purification system connected to an Elix3 pre-system (Millipore, Billerica, MA) was used to prepare the mobile phase and sample solutions. Phenylhydrazine (>98\% purity) and formic acid (LC-MS grade) were purchased from Tokyo Chemical Industry (Tokyo, Japan). A supply of 3-iodophenyl isocyanate ( $>97 \%$ purity) was purchased from Sigma-Aldrich (Milwaukee, WI). Supplies of 2,3,5-triiodobenzoyl chloride ( $>96 \%$ purity), HPLC-grade methanol and acetonitrile (ACN), and organic synthesis-grade pyridine were purchased from Wako Pure Chemical Industries (Osaka, Japan). Special grade tetrahydrofuran (THF) and $\mathrm{N}, \mathrm{N}$-dimethylformamide (DMF) were purchased from Kanto Chemical (Tokyo, Japan). Argon (Ar) gas (>99.999\% purity) and oxygen $\left(\mathrm{O}_{2}\right)$ gas $(>99.999 \%$ purity) were purchased from Taiyo Nippon Sanso (Tokyo, Japan). Five lots of antipyrine (an analgesic antipyretic drug) were purchased as follows: one lot of extra pure grade ( $>98.0 \%$ purity) from Kanto Chemical, two lots of first grade (>99.0\%) from Tokyo Chemical Industry, and two lots of biochemistry grade $(>99.0 \%)$ from Wako Pure Chemical Industries.

\section{Derivatization reaction}

The derivatization schemes for the two derivatization reagents are shown in Fig. 1. For 3-iodophenyl isocyanate derivatization, $20 \mu \mathrm{L}$ of 3-iodophenyl isocyanate was diluted with THF to $10 \mathrm{~mL}$, which was then used as the derivatization solution. The derivatization was carried out by adding $0.5 \mathrm{~mL}$ of the derivatization solution to $1 \mathrm{~mL}$ of THF containing approximately $25 \mathrm{mg}$ of antipyrine. After sonication at room temperature, the solution was diluted with $2 \mathrm{~mL}$ of DMF and an $80 \%$ ACN aqueous solution to $5 \mathrm{~mL}$.

For 2,3,5-triiodobenzoyl chloride derivatization, the derivatization solution was prepared using $20 \mathrm{mg}$ of 2,3,5-triiodobenzoyl chloride in $10 \mathrm{~mL}$ of THF and $0.5 \mathrm{~mL}$ of pyridine. After sonication for $5 \mathrm{~min}$ at $50^{\circ} \mathrm{C}$, this solution was diluted with THF to $20 \mathrm{~mL}$. The derivatization was carried out

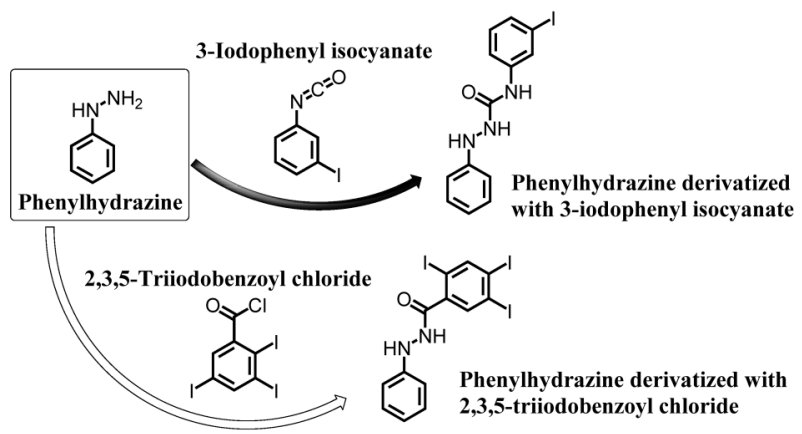

Fig. 1 Derivatization scheme for LC-ICP-MS analysis.

Table 1 ICP-MS conditions

\begin{tabular}{ll}
\hline \multicolumn{1}{c}{ Parameter } & \multicolumn{1}{c}{ Value } \\
\hline Mass/Analyte & $\mathrm{m} / \mathrm{z} 127$ (iodine) \\
RF power & $1600 \mathrm{~W}$ \\
Plasma gas (Ar) flow rate & $17 \mathrm{~L} / \mathrm{min}$ \\
Auxiliary gas (Ar) flow rate & $1.2 \mathrm{~L} / \mathrm{min}$ \\
Nebulizer gas (Ar) flow rate & $0.98 \mathrm{~L} / \mathrm{min}$ \\
Ion lens & $7.8(\mathrm{~V})$ \\
Sample introduction gas $\left(\mathrm{O}_{2}\right)$ flow rate & $30 \mathrm{~mL} / \mathrm{min}$ \\
Integration time & $40 \mathrm{~min}$ \\
\hline
\end{tabular}

by adding $0.5 \mathrm{~mL}$ of the derivatization solution to $1 \mathrm{~mL}$ of THF containing approximately $25 \mathrm{mg}$ of antipyrine. After sonication at $50^{\circ} \mathrm{C}$, the solution was diluted with $2 \mathrm{~mL}$ of $\mathrm{DMF}$ and an $80 \% \mathrm{ACN}$ aqueous solution to $5 \mathrm{~mL}$.

\section{LC-ICP-MS system}

An ELAN DRC II ICP-MS instrument (PerkinElmer, Waltham, MA) was used in this study. A platinum cone, quartz injector (2.0 $\mathrm{mm}$ orifice), PC3 spray chamber, and MEINEHARD glass nebulizer were used with the instrument. The mobile phase was connected to the glass nebulizer. The PC3 spray chamber was cooled to $5^{\circ} \mathrm{C}$, and an introduction gas $\left(\mathrm{O}_{2}\right)$ was used. The $\mathrm{O}_{2}$ gas prevents buildups of carbon on the interface cones and leads to a stable ion intensity. The ICP-MS conditions are summarized in Table 1.

A two-dimensional LC (2D-LC) system (column switching system) was employed to separate the analyte from interferences. The HPLC gradient system (the first LC system for separation) consisted of two LC-20AD pumps, an SIL-20AC autosampler, and a CTO-20AC column oven (Shimadzu, Kyoto, Japan). In addition, two LC-20AD pumps were used for the second HPLC gradient system (the second LC system for analysis). The HPLC instrumental setup was the same as in the previous study. ${ }^{28}$

An L-column C8 $(3 \mu \mathrm{m}, 2.0 \mathrm{~mm}$ i.d. $\times 150 \mathrm{~mm}$, CERI, Tokyo, Japan) (for the first LC system) and an InertSustain C18 (3 $\mu \mathrm{m}$, $2.1 \mathrm{~mm}$ i.d. $\times 250 \mathrm{~mm}$, GL Sciences, Tokyo, Japan) (for the second LC system) column were used for the analysis of the 3-iodophenyl isocyanate derivative. An InertSustain C18 (3 $\mu \mathrm{m}$, $2.1 \mathrm{~mm}$ i.d. $\times 150 \mathrm{~mm}$ ) (for the first LC system) and InertSustain C18 $(3 \mu \mathrm{m}, 2.1 \mathrm{~mm}$ i.d. $\times 250 \mathrm{~mm}$ ) columns (for the second LC system) were used for the analysis of the 2,3,5-triiodobenzoyl chloride derivative. The flow rate of the mobile phase was set at $0.2 \mathrm{~mL} / \mathrm{min}$. The column temperature was maintained at $40^{\circ} \mathrm{C}$. A $10-\mu \mathrm{L}$ aliquot of either the sample solution or standard 
solution was injected. Derivatized compounds were monitored by $m / z, 127$ (iodine).

\section{LC-MS system}

An Orbitrap MS (Thermo Fisher Scientific, Waltham, MA) equipped with an LC-30AD two-dimensional LC system (Shimadzu) was used to confirm the structure of the derivatized compound of phenylhydrazine. The Orbitrap MS was operated in the ESI positive mode with a sheath gas flow of $50 \mathrm{arb}$, an aux gas flow of $10 \mathrm{arb}$, a spray voltage of $5.0 \mathrm{kV}$, a capillary temperature of $300^{\circ} \mathrm{C}$, a capillary voltage of $30 \mathrm{~V}$, and a tube lens voltage of $10 \mathrm{~V}$.

Preparation of standard and sample solutions for method validation

The standard stock solution was prepared at approximately $50 \mathrm{ppm}(\mu \mathrm{g} / \mathrm{g}$ API) (relative to $25 \mathrm{mg}$ of API), which corresponds to a $0.25 \mu \mathrm{g} / \mathrm{mL}$ phenylhydrazine solution. The standard stock solution was diluted to $0.06,0.08,0.1,0.2,0.5,1$, and $10 \mathrm{ppm}$ with an $80 \%$ ACN aqueous solution. These solutions were used to estimate the linearity, DL, and quantitation limit (QL) validation $(0.06$ and $0.08 \mathrm{ppm}$ solutions were prepared only for the 2,3,5-triiodobenzoyl chloride derivative).

The sample solution was prepared using approximately $25 \mathrm{mg}$ of antipyrine. Blank solutions were also prepared without antipyrine using the same procedure. The sample solutions spiked with 1,10 , and $50 \mathrm{ppm}$ of phenylhydrazine were prepared in triplicate. The standard solution was prepared at $10 \mathrm{ppm}$. These solutions were used for method validation of specificity, accuracy, and precision.

\section{Method validation}

The linearity was validated using $0.1,0.2,0.5,1,10$, and $50 \mathrm{ppm}$ solutions. The DL and QL were based on the relative standard deviation (RSD) of the peak area obtained from six injections of the 3-iodophenyl isocyanate derivative ( 0.2 and $0.5 \mathrm{ppm}$ ) and 2,3,5-triiodobenzoyl chloride derivative (0.06 and $0.2 \mathrm{ppm})$. The specificity was validated using a blank solution, standard solution with $10 \mathrm{ppm}$ phenylhydrazine, sample solution, and sample solution spiked with $1 \mathrm{ppm}$ of phenylhydrazine. The accuracy (recovery) was validated using the standard solution $(10 \mathrm{ppm})$ and sample solution spiked with three different concentrations $(1,10$, and $50 \mathrm{ppm})$. The standard and sample solutions were analyzed in triplicate. The repeatability of the spike recovery (three repetitions at three concentrations) was confirmed for the precision validation. The range was validated by the results for linearity, accuracy, and precision.

Quantification of phenylhydrazine in antipyrine by LC-ICP-MS

For the 3-iodophenyl isocyanate derivatization method, approximately $25 \mathrm{mg}$ of antipyrine was weighed and dissolved in $1 \mathrm{~mL}$ of $\mathrm{THF}$, and a $0.5-\mathrm{mL}$ aliquot of the derivatization solution was added to the solution. After incubation at room temperature for $15 \mathrm{~min}$ with sonication, the solution was diluted to $5 \mathrm{~mL}$ with $2 \mathrm{~mL}$ of $\mathrm{DMF}$ and an $80 \% \mathrm{ACN}$ aqueous solution, and this was used as the sample solution. A standard solution was separately prepared following the same procedure using $1 \mathrm{~mL}$ of a $0.25-\mu \mathrm{g} / \mathrm{mL}$ phenylhydrazine THF solution.

For the 2,3,5-triiodobenzoyl chloride derivatization method, approximately $25 \mathrm{mg}$ of antipyrine was weighed and dissolved in $1 \mathrm{~mL}$ of $\mathrm{THF}$, and a $0.5-\mathrm{mL}$ aliquot of the derivatization solution was added to the solution. After incubation at $50^{\circ} \mathrm{C}$ for 15 min with sonication, the solution was diluted to $5 \mathrm{~mL}$ with $2 \mathrm{~mL}$ of DMF and an $80 \% \mathrm{ACN}$ aqueous solution, and this was used as the sample solution. A standard solution was separately prepared following the same procedure using $1 \mathrm{~mL}$ of a $0.25-\mu \mathrm{g} / \mathrm{mL}$ phenylhydrazine THF solution.

\section{Results and Discussion}

\section{D-LC for ICP-MS}

A two-dimensional LC system (column switching system) was employed to separate the analyte from the matrix, i.e., API and the derivatization reagent in the sample solution. In the first LC system, the analyte was separated from the matrix, and the fraction including the analyte was directly introduced into the second LC system by switching the valves. Under optimized conditions, a baseline separation of the interferences and analyte could be achieved, and the analysis was conducted in $40 \mathrm{~min}$. The optimized condition for 3-iodophenyl isocyanate and 2,3,5-triiodobenzoyl chloride are summarized in Tables 2 and 3, respectively.

\section{Derivatization with 3-iodophenyl isocyanate (mono-iodo} derivatization method)

The derivatization conditions were optimized in the following experiments. The progress of the reaction in ACN and THF was investigated to select the best 3-iodophenyl isocyanate reaction solvent. Since the reaction efficiencies were almost the same, both solvents could be used for the reaction. In this study, THF was selected as the reaction solvent and used in the following experiments. The sonication temperature was tested at room temperature and $50^{\circ} \mathrm{C}$. The derivatization reaction was complete even at room temperature. Incubation times of 5, 15, and 30 min were tested, and the derivatization reaction was complete within $15 \mathrm{~min}$. Therefore, incubation for $15 \mathrm{~min}$ at room temperature was selected for the derivatization reaction.

To confirm the structure of the 3-iodophenyl isocyanate derivative, structural analysis was conducted using LC-MS. The protonated ion $[\mathrm{M}+\mathrm{H}]^{+}$at $\mathrm{m} / \mathrm{z}, 354.010$ and fragment ions at $\mathrm{m} / \mathrm{z}$ 336.984 and $\mathrm{m} / \mathrm{z} 219.962$ were detected, and the results suggested the structure of the phenylhydrazine derivatized with 3-iodophenyl isocyanate (Fig. 2).

\section{Validation results of mono-iodo derivatization method}

The specificity was confirmed using a blank solution, $10 \mathrm{ppm}$ phenylhydrazine standard solution, sample solution, and sample solution spiked with $1 \mathrm{ppm}$ of phenylhydrazine. In the chromatogram of the sample solution, no peak was observed around the retention time of the phenylhydrazine derivatized with 3-iodophenyl isocyanate. Linearity in the 0.5 to $50 \mathrm{ppm}$ range was obtained, and the $R^{2}$ value was 0.9998 . The relative standard deviations (RSDs) of the six injections of the 0.2 and $0.5 \mathrm{ppm}$ solutions were 3.4 and $6.1 \%$, respectively. Thus, the DL and QL were 0.2 and $0.5 \mathrm{ppm}$, respectively, which corresponded to 0.95 and $2.38 \mathrm{ng} / \mathrm{mL}$ of phenylhydrazine. The accuracy (recovery test) was in the range of 99.5 to $111.9 \%$ at 1 to $50 \mathrm{ppm}$. The repeatability of the spike recovery test was $3.7 \%$, and good spike recovery and repeatability were obtained. The linearity, accuracy, and precision results support a range from 1 to $50 \mathrm{ppm}$. This LC-ICP-MS method using 3-iodophenyl isocyanate offers a method for the analysis of phenylhydrazine in antipyrine at sub-ppm levels.

\section{Derivatization with 2,3,5-triiodobenzoyl chloride (tri-iodo derivatization method)}

As described above, the LC-ICP-MS method using 3 -iodophenyl isocyanate has sufficient sensitivity. However, if 
Table 2 HPLC conditions for 3-iodophenyl isocyanate derivatization method

\begin{tabular}{|c|c|c|c|}
\hline \multicolumn{4}{|c|}{ Separation condition (First LC system) } \\
\hline Column & \multicolumn{2}{|c|}{ L-column C8 } & $(3 \mu \mathrm{m}, 2.0 \mathrm{~mm}$ i.d. $\times 150 \mathrm{~mm}, \mathrm{CERI})$ \\
\hline Column temperature & \multicolumn{3}{|c|}{$40^{\circ} \mathrm{C}$} \\
\hline Flow rate & \multicolumn{3}{|l|}{$0.2 \mathrm{~mL} / \mathrm{min}$} \\
\hline Injection volume & \multicolumn{3}{|l|}{$10 \mu \mathrm{L}$} \\
\hline Mobile phase A & \multicolumn{3}{|c|}{$0.05 \%$ Formic acid in water } \\
\hline Mobile phase B & \multicolumn{3}{|c|}{$0.05 \%$ Formic acid in acetonitrile } \\
\hline \multirow[t]{3}{*}{ Gradient program } & Time/min & $\mathbf{A \%}$ & В\% \\
\hline & 0 & 75 & 25 \\
\hline & 30 & 10 & 90 \\
\hline \multirow[t]{4}{*}{ Valve program } & Time/min & Valve pos & \\
\hline & 0 & A & \\
\hline & 15.9 & B & \\
\hline & 16.4 & A & \\
\hline
\end{tabular}

Analytical condition (Second LC system)

Analytical column InertSustain C18

$(3 \mu \mathrm{m}, 2.1 \mathrm{~mm}$ i.d. $\times 250 \mathrm{~mm}$, GL Sciences $)$

Column temperature $40^{\circ} \mathrm{C}$

$\begin{array}{ll}\text { Flow rate } & 0.2 \mathrm{~mL} / \mathrm{min} \\ \text { Mobile phase C } & \text { Water }\end{array}$

Mobile phase D Methanol

Gradient program Time/min $\begin{array}{ll}\mathbf{A} \% & \mathbf{B} \%\end{array}$ $\begin{array}{lll}0.4 & 40 & 60 \\ 16.4 & 40 & 60\end{array}$

$40 \quad 10 \quad 90$

\section{Mass Spectra}

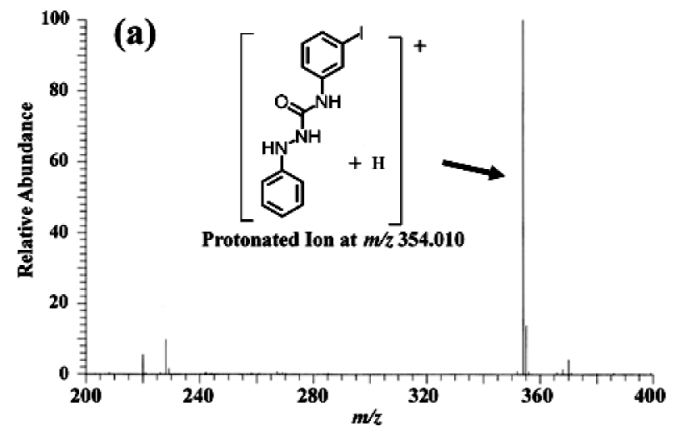

Mass/Mass spectra

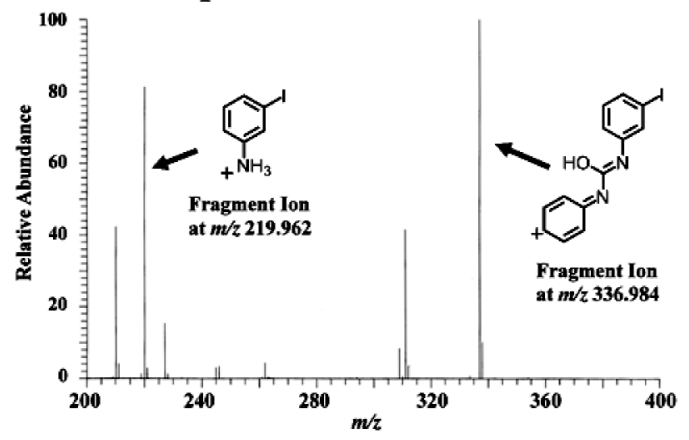

Table 3 HPLC conditions for 2,3,5-triiodobenzoyl chloride derivatization method

Separation condition (First LC system)

Column InertSustain C18

Column temperature $40^{\circ} \mathrm{C}$

$3 \mu \mathrm{m}, 2.1 \mathrm{~mm}$ i.d. $\times 150 \mathrm{~mm}, \mathrm{GL}$ Sciences $)$

Flow rate

$0.2 \mathrm{~mL} / \mathrm{min}$

Injection volume $\quad 10 \mu \mathrm{L}$

Mobile phase A $\quad 0.05 \%$ Formic acid in water

Mobile phase B

$0.05 \%$ Formic acid in acetonitrile

Gradient program

Time/min A\%

$\begin{array}{lll}30 & 30 & 30 \\ & 10 & 90\end{array}$

Valve program

Time/min Valve position

$\begin{array}{cc}0 & \text { A } \\ 19.4 & \text { B }\end{array}$

19.9

\begin{tabular}{|c|c|c|c|}
\hline \multicolumn{4}{|c|}{ Analytical condition (Second LC system) } \\
\hline Analytical column & \multicolumn{3}{|c|}{$\begin{array}{l}\text { InertSustain C18 } \\
(3 \mu \mathrm{m}, 2.1 \mathrm{~mm} \text { i.d. } \times 250 \mathrm{~mm} \text {, GL Sciences })\end{array}$} \\
\hline Column temperature & \multicolumn{3}{|c|}{$40^{\circ} \mathrm{C}$} \\
\hline Flow rate & \multicolumn{3}{|l|}{$0.2 \mathrm{~mL} / \mathrm{min}$} \\
\hline Mobile phase $\mathrm{C}$ & \multicolumn{3}{|l|}{ Water } \\
\hline Mobile phase D & \multicolumn{3}{|l|}{ Methanol } \\
\hline \multirow[t]{4}{*}{ Gradient program } & Time/min & A\% & B\% \\
\hline & 0 & 40 & 60 \\
\hline & 19.9 & 40 & 60 \\
\hline & 40 & 10 & 90 \\
\hline
\end{tabular}

\section{Mass Spectra}

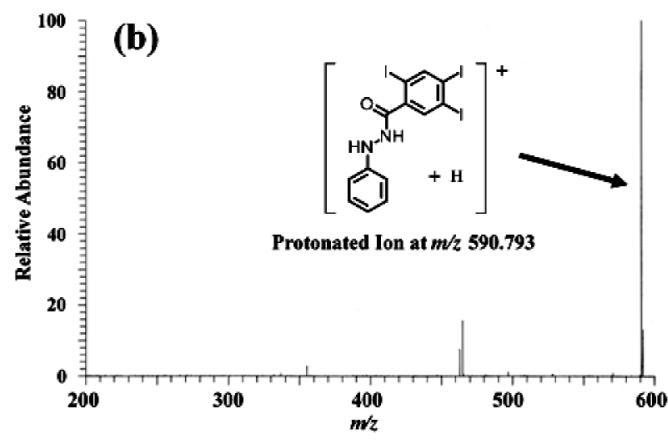

Mass/Mass spectra

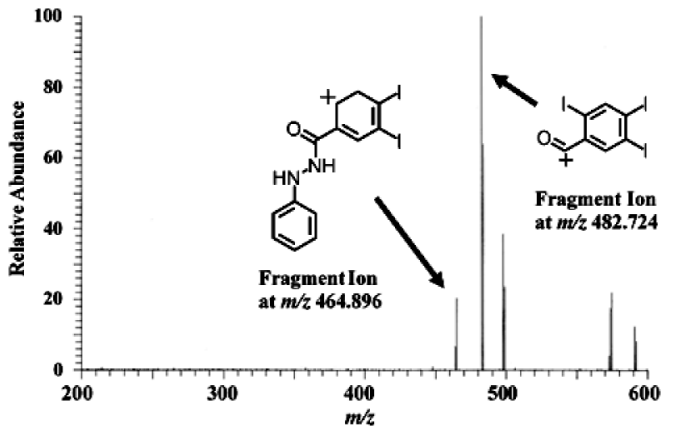

Fig. 2 Structural analysis of the iodo derivative by LC-MS. The structure of the iodo derivatized phenylhydrazine was confirmed by the protonated ion and the fragment ions. Mass and mass/mass spectra are shown in (a) 3-iodophenyl isocyanate derivative and (b) 2,3,5-triiodobenzoyl chloride derivative.

derivatization reagents with multiple iodine atoms are employed, the DL and QL value might be improved because of the increase in the number of target atoms (iodine). In this respect, 2,3,5-triiodobenzoyl chloride, which has three iodine atoms in its molecule, was also investigated as a derivatization reagent.

THF was used as the reaction solvent, and the reaction 

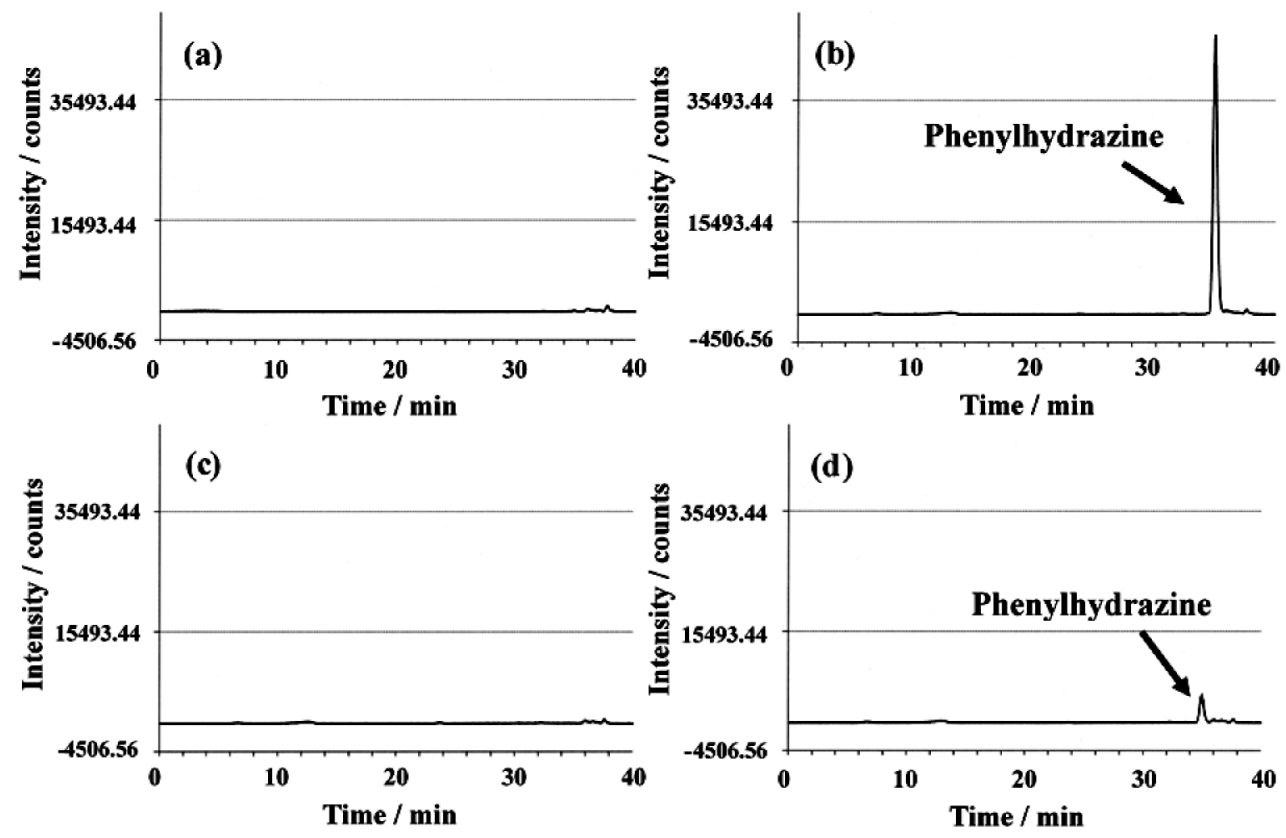

Fig. 3 LC-ICP-MS chromatogram of phenylhydrazine derivatized with 2,3,5-triiodobenzoyl chloride for specificity. The specificity was confirmed using (a) a blank solution, (b) a standard solution (10 ppm), (c) a sample solution, and (d) a sample solution spiked with phenylhydrazine (1 ppm).

conditions were optimized in the following experiments. The sonication temperature was tested at room temperature, 50 , and $70^{\circ} \mathrm{C}$. Since the derivatization reaction was complete at $50^{\circ} \mathrm{C}$, the reaction temperature was set at $50^{\circ} \mathrm{C}$. Then, incubation times of $5,15,30$, and $60 \mathrm{~min}$ were tested. Though the derivatization reaction was complete within $5 \mathrm{~min}$, the incubation time was set to $15 \mathrm{~min}$ to guarantee a complete reaction.

To confirm the structure of the 2,3,5-triiodobenzoyl chloride derivative, structural analysis was conducted using LC-MS. The protonated ion $[\mathrm{M}+\mathrm{H}]^{+}$at $\mathrm{m} / \mathrm{z} 590.793$ and the fragment ions at $\mathrm{m} / \mathrm{z} 482.724$ and $\mathrm{m} / \mathrm{z} 464.896$ were detected, and the results suggested the structure of the phenylhydrazine derivatized with 2,3,5-triiodobenzoyl chloride (Fig. 2).

\section{Validation results of tri-iodo derivatization method}

The specificity was confirmed using a blank solution, $10 \mathrm{ppm}$ phenylhydrazine standard solution, sample solution, and sample solution spiked with $1 \mathrm{ppm}$ of phenylhydrazine. In the chromatogram of the sample solution, no peak was observed around the retention time of the phenylhydrazine derivatized with 2,3,5-triiodobenzoyl chloride (Fig. 3). Linearity in the 0.2 to $50 \mathrm{ppm}$ range was obtained, and the $R^{2}$ value was 0.9983 . The RSD of the six injections of the 0.06 and $0.2 \mathrm{ppm}$ solutions were 6.7 and $2.6 \%$, respectively. Thus, the DL and QL were 0.06 and $0.2 \mathrm{ppm}$, respectively, which corresponds to 0.29 and $0.97 \mathrm{ng} / \mathrm{mL}$ of phenylhydrazine. The accuracy (recovery test) was in the range of 99.1 to $111.1 \%$ at 1 to $50 \mathrm{ppm}$ (Table 4), and the repeatability of the spike recovery test was $4.0 \%$. The linearity, accuracy, and precision results support a range from 1 to $50 \mathrm{ppm}$. The spike recovery rates were about $100 \%$ even at $1 \mathrm{ppm}$. Considering that the desired sensitivity for GTI analysis is at the sub-ppm level, the developed method was successfully applied for the phenylhydrazine analysis of antipyrine.

Quantification of phenylhydrazine in antipyrine by LC-ICP-MS

Because phenylhydrazine is commonly used as a raw material
Table 4 Accuracy for 2,3,5-triiodobenzoyl chloride

derivatization method

\begin{tabular}{lccc}
\hline \multirow{2}{*}{ Accuracy } & \multicolumn{3}{c}{ Recovery, \% } \\
\cline { 2 - 4 } & $n=1$ & $n=2$ & $n=3$ \\
\hline Spiked with 1 ppm & 100.9 & 100.9 & 99.1 \\
Spiked with 10 ppm & 106.8 & 105.7 & 106.3 \\
Spiked with 50 ppm & 109.6 & 109.0 & 111.1 \\
Average, \% & & 105.5 & 4.27 \\
Standard Deviation, \% & & 4.0 \\
Relative Standard Deviation, \% & & 4 \\
\hline
\end{tabular}

for antipyrine, residual phenylhydrazine may present in antipyrine. The residual amounts of phenylhydrazine in five different commercially available lots of antipyrine were analyzed by the developed methods using 3-iodophenyl isocyanate or 2,3,5-triiodobenzoyl chloride as the derivatization reagent. The results showed that the phenylhydrazine residues in all the lots were less than each DL of 0.2 and $0.06 \mathrm{ppm}$.

In this study, the acyl halide and an isocyanate compound containing iodine were employed as the derivatization reagents for phenylhydrazine analysis. First, 3-iodophenyl isocyanate, which has one iodine atom, was investigated as the derivatization reagent. The developed method gave excellent method validation results in the range of 1 to $50 \mathrm{ppm}$ relative to the API. Then, 2,3,5-triiodobenzoyl chloride, which has three iodine atoms, was also investigated as a derivatization reagent to enhance the sensitivity of ICP-MS detection. As expected, the $\mathrm{DL}$ of the method was $0.29 \mathrm{ng} / \mathrm{mL}$ of phenylhydrazine solution, and this value was one third of that obtained from the 3-iodophenyl isocyanate method $(0.95 \mathrm{ng} / \mathrm{mL})$. This data strongly indicate the contribution of the number of iodine atoms to the sensitivity. This method gave excellent method validation results in the range of 1 to $50 \mathrm{ppm}$ relative to the API. 
Furthermore, five lots of antipyrine were analyzed by the developed methods using 3-iodophenyl isocyanate or 2,3,5-triiodobenzoyl chloride, and all the results were less than each DL of 0.2 and $0.06 \mathrm{ppm}$.

Concerning the analysis of GTIs in APIs, David et al. have conducted some studies to develop a strategic approach for the analysis, and reported the decision tree on the method selection for GTI analysis. ${ }^{10}$ In their article, the LC-MS method using hexylchloroformate derivatization was developed as an analytical method for phenylhydrazine in APIs. The sample solution was prepared in a concentration of $100 \mathrm{mg} / \mathrm{mL}$, and the linearity in the 0.25 to $5 \mathrm{ppm}$ range was obtained with an $R^{2}$ value of $>0.99$. A comparison of the range of developed LC-ICP-MS methods to that of the LC-MS method showed that the sensitivity of the developed methods is either the same or better than that of the LC-MS method.

This proves that the LC-ICP-MS methods allow for the analysis of phenylhydrazine at sub-ppm levels. The methods can be used for batch release testing, and they are also applicable to the process control testing of API manufacturing. Moreover, the methods can be applied to the analysis of GTIs containing a hydrazino, amino and/or hydroxyl group in the molecule.

\section{Conclusions}

Sensitive quantitation methods using LC-ICP-MS with iododerivatization were developed. The methods allow for the trace level analysis of phenylhydrazine in antipyrine. The QL values of the methods were sufficient for sub-ppm analysis.

\section{References}

1. The International Conference on Harmonization, ICH Q3A/B, "Impurities in New Drug Substances/New Drug Products".

2. US FDA, Guidance for Industry: Genotoxic and Carcinogenic Impurities in Drug Substances and Products: Recommended Approaches. Draft, December, 2008.

3. EMA, Guideline on the limits of genotoxic impurities, CPMP/SWP/5199/02， EMEA/CHMP/QWP/251344/2006, 28 June, 2006.

4. EMA, Questions and Answers on the Guideline on the limits of genotoxic impurities, EMEA/CHMP/SWP/ 431994/2007, 23 September, 2010.

5. L. Müller, R. J. Mauthe, C. M. Riley, M. M. Andino, D. De Antonis, C. Beels, J. DeGeorge, A. G. M. De Knaep, D. Ellison, J. A. Fagerland, R. Frank, B. Fritschel, S. Galloway, E. Harpur, C. D. N. Humfrey, A. S. Jacks, N. Jagota, J. Mackinnon, G. Mohan, D. K. Ness, M. R. O’Donovan, M.
D. Smith, G. Vudathala, and L. Yotti, Regul. Toxicol. Pharmacol., 2006, 44, 198.

6. C. M. Callis, J. P. Bercu, K. M. DeVries, L. K. Dow, D. K. Robbins, and D. L. Varie, Org. Process Res. Dev., 2010, 14, 986.

7. D. Kirkland and D. Snodin, Int. J. Pharm. Med., 2004, 18, 197.

8. D. I. Robinson, Org. Process Res. Dev., 2010, 14, 946.

9. N. V. V. S. S. Raman, A. V. S. S. Prasad, and K. Ratnakar Reddy, J. Pharm. Biomed. Anal., 2011, 55, 662.

10. A. Teasdale, "Genotoxic Impurities: Strategies for Identification and Control", 2010, John Wiley and Sons, Hoboken, NJ.

11. D. P. Elder, A. M. Lipczynski, and A. Teasdale, J. Pharm. Biomed. Anal., 2008, 48, 497.

12. D. Q. Liu, M. Sun, and A. S. Kord, J. Pharm. Biomed. Anal., 2010, 51, 999.

13. Z. Y. Yuabova, D. R. Holschlag, S. A. Rodriguez, C. Qin, V. V. Papov, F. Qiu, J. F. McCaffrey, and D. L. Norwood, J. Liq. Chromatogr. Relat. Technol., 2008, 31, 2318.

14. G. Vanhoenacker, E. Dumont, F. David, A. Baker, and P. Sandra, J. Chromatogr. A, 2009, 1216, 3563.

15. J. An, M. Sun, L. Bai, T. Chen, D. Q. Liu, and A. Kord, J. Pharm. Biomed. Anal., 2008, 48, 1006.

16. L. Bai, M. Sun, J. An, D. Q. Liu, T. Chen, and A. S. Kord, J. Chromatogr. A, 2010, 1217, 302.

17. M. Sun, L. Bai, and D. Q. Liu, J. Pharm. Biomed. Anal., 2009, 49, 529.

18. F. David, K. Jacq, P. Sandra, A. Baker, and M. S. Klee, Anal. Bioanal. Chem., 2010, 396, 1291.

19. H. E. Strohmeyer and G. W. Sluggett, J. Pharm. Biomed. Anal., 2012, 62, 216.

20. K. Harigaya, H. Yamada, K. Yaku, H. Nishi, and J. Haginaka, Chem. Pharm. Bull., 2014, 62, 395.

21. K. Harigaya, Y. Kuwahara, and H. Nishi, Chem. Pharm. Bull., 2008, 56, 475.

22. J. E. Carr, A. E. Dill, K. Kwok, J. W. Carnahan, and G. K. Webster, Curr. Pharm. Anal., 2008, 4, 206.

23. A. S. Pereira, M. Schelfaut, F. Lynen, and P. Sandra, J. Chromatogr. A, 2008, 1185, 78.

24. G. K. Zoorob, J. W. McKiernan, and J. A. Caruso, Mikrochim. Acta, 1998, 128, 145.

25. R. Nageswara Rao and M V. N. Kumar Talluri, J. Pharm. Biomed. Anal., 2007, 43, 1.

26. G. Koellensperger, M. Groeger, D. Zinkl, P. Petzelbauer, and S. Hann, J. Anal. At. Spectrom., 2009, 24, 97.

27. D. Iwahata, K. Hirayama, and H. Miyano, J. Anal. At. Spectrom., 2008, 23, 1063.

28. K. Harigaya, H. Yamada, K. Yaku, H. Nishi, and J. Haginaka, Anal. Sci., 2014, 30, 377. 